\title{
Aspectos epidemiológicos dos pólipos e lesões plano-elevadas colorretais
}

\author{
Epidemiological aspects of colorectal polyps and flat adenomas
}

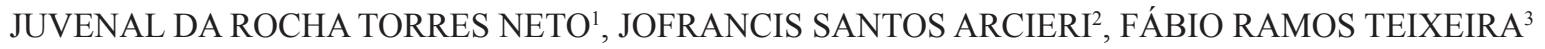

\begin{abstract}
${ }^{1}$ Chefe do Serviço de Coloproctologia da Universidade Federal de Sergipe (UFS), Professor Doutor e Coordenador do internato de Clínica Cirúrgica. ${ }^{2}$ Médica Residente de Clínica Médica do Hospital de Urgência de Sergipe Governador João Alves Filho. ${ }^{3}$ Médico Residente de Coloproctologia do Hospital Universitário da UFS - São Cristóvão (SE), Brasil.
\end{abstract}

TORRES NETO JR; ARCIERI JS; TEIXEIRA FR. Aspectos epidemiológicos dos pólipos e lesões plano-elevadas colorretais. Rev bras Coloproct, 2011;30(4): 419-429.

RESUMO: Introdução: Os pólipos e as lesões plano-elevadas colorretais são importantes na prevenção do câncer colorretal pelo risco de malignização dos adenomas. Objetivo: traçar o perfil demográfico dos pacientes com diagnóstico endoscópico de pólipos e/ou lesões plano-elevadas colorretais no Hospital Universitário da Universidade Federal de Sergipe e Torres Centro Médico. Metodos: Foram avaliados 6.919 prontuários de 2002 a 2007, enfatizando-se as variáveis: idade, gênero, procedência e indicação; número, tamanho, morfologia, histologia, grau de displasia, topografia, lesões sincrônicas e metacrônicas, e diagnósticos associados. Resultados: Foram encontradas $1.031(13,51 \%)$ lesões em 935 exames, correspondendo a 826 pacientes, $46 \%$ masculino e 54\% feminino. A idade variou de 3 a 96 anos, com média de 53,64. A distribuição topográfica mais frequente das lesões polipoides foi em reto e sigmoide (58,40\%). As lesões eram sésseis em $\mathbf{5 2 , 8 0 \%}$, pediculadas em 27,90\% e plano-elevadas em 19,30\% dos casos. Ocorreram lesões sincrônicas em $\mathbf{2 3 , 4 8 \%}$ e metacrônicas em 30,10\% dos pacientes. Histologicamente, 43,36\% eram adenomas, sendo 85,70\% tubulares, 9,60\% tubulovilosos e 4,70\% vilosos; $30,64 \%$ eram pólipos hiperplásicos, $15,80 \%$ inflamatórios e 10,20\% possuíam outros tipos histológicos. Os adenomas apresentavam displasia de baixo grau em $83,40 \%$ dos casos e alto grau em $16,60 \%$. Sete eram adenocarcinomas, um carcinoide e um tumor gastrointestinal estromal. Conclusões: A colonoscopia e a polipectomia são importantes no diagnóstico e prevenção do câncer colorretal.

Descritores: Adenoma. Pólipos. Colonoscopia.

\section{INTRODUÇÃO}

Os pólipos colorretais são estruturas que se projetam na superfície da camada mucosa do intestino grosso, podendo ser neoplásicos ou não ${ }^{1}$.

Foi Morson, em 1976, quem melhor estabeleceu uma classificação para os diversos tipos de pólipos e a importância da progressão adenoma-câncer. Os pólipos foram divididos em: pólipos neoplásicos, caracterizados pelos adenomas e os carcinomas, e os pólipos não-neoplásicos, que incluem os tipos epiteliais hamartomatosos, inflamatórios, hiperplásicos ou metaplásicos ${ }^{2}$.
Os pólipos adenomatosos, que correspondem a cerca de $70 \%$ de todos os pólipos, são conhecidamente lesões pré-malignas que antecedem, em 10 a 15 anos, o câncer colorretal ${ }^{3,4}$. Por conta dessa progressão lenta, a detecção de lesões pré-neoplásicas no intestino grosso é relevante na prevenção do surgimento e complicações do câncer colorretal. Os pólipos podem ser ressecados antes da sua malignização, diminuindo sobremaneira a taxa de morbimortalidade do câncer colorretal.

A Japanese Research Society for Cancer of the Colon and Rectum classifica adenomas e cânceres colorretais em dois grupos: protruso e superficial. O tipo

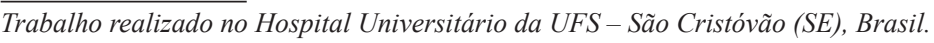

$\overline{\text { Recebido em 20/08/2010 }}$

Aceito para publicação em 06/10/2010 
protruso são os pólipos colorretais. O tipo superficial são as lesões não-polipoides colorretais, classificadas em superficial elevada, plana e deprimida 5 .

A colonoscopia é o padrão-ouro para o diagnóstico do câncer colorretal e para a detecção e ressecção endoscópica das lesões precursoras. A realização de polipectomias e biópsias permite, através da histopatologia, avaliar o tipo histológico, o grau de displasia e as margens de ressecção a fim de quantificar seu potencial de malignização ${ }^{1,6,7}$.

O câncer colorretal é a quinta neoplasia maligna mais frequente no Brasil, e se estima que 26.990 novos casos tenham sido diagnosticados no ano de 2008, o que evidencia sua alta frequência ${ }^{8}$. Estes valores correspondem a um risco estimado de 13 casos novos a cada 100 mil homens e 15 para cada 100 mil mulheres. Associado a isso, a grande maioria dos tumores malignos se origina dos adenomas, e a detecção e retirada precoces evitam a progressão para o câncer. Estes dados motivaram a realização deste trabalho no sentido de analisar retrospectivamente as seguintes variáveis: dados gerais do paciente como idade, gênero, procedência e indicação do exame; dados da lesão polipoide como frequência, distribuição topográfica, aspecto morfológico, tipo histológico, grau de displasia, tamanho, presença de lesões sincrônicas e/ou metacrônicas e ocorrência de diagnósticos associados.

\section{PACIENTES E MÉTODOS}

Trata-se de estudo retrospectivo de corte transversal, observacional e descritivo, para avaliar o perfil dos pacientes com pólipos e/ou lesões plano-elevadas colorretais diagnosticadas nos Serviços de Vídeocolonoscopia do Hospital Universitário da Universidade Federal de Sergipe (HU/UFS) e do Torres Centro Médico, no período de janeiro de 2002 a dezembro de 2007, a partir da análise de prontuários. Para tanto, foi feito um levantamento de todos os laudos de retossigmoidoscopia e colonoscopia no referido período, sendo selecionados os pacientes que apresentaram pólipos e/ou lesões plano-elevadas, segundo descrição diagnóstica do endoscopista e, em seguida, avaliados os seus exames anatomopatológicos.

A partir da análise dos laudos endoscópicos e histopatológicos, foram obtidas as seguintes variáveis: dados gerais do paciente (idade, gênero, procedência e indicação do exame); dados da lesão polipoide (número, tamanho, aspecto morfológico, tipo histológico, grau de displasia, distribuição topográfica, presença de lesões sincrônicas e/ou metacrônicas); ocorrência de diagnósticos associados (Anexo 1).

Os dados foram inicialmente coletados dos prontuários médicos e registrados em arquivo informatizado em tabulação das variáveis por ano. Não houve padronização na aplicação dos critérios classificatórios pelos endoscopistas e patologistas, sendo mantida a variabilidade existente na rotina do Serviço.

Foram considerados critérios de exclusão: pacientes com diagnóstico de polipose intestinal, pólipos não ressecados ou biopsiados, pólipos cujo exame anatomopatológico foi extraviado.

As displasias foram classificadas em baixo grau e alto grau, conforme orientação da Organização Mundial de Saúde (OMS) ${ }^{9}$. As displasias classificadas em atipia leve foram enquadradas como baixo grau, e as displasias classificadas em atipia moderada e severa como alto grau. Os adenomas que não referiam o grau de displasia foram classificados como displasia de baixo grau. As lesões malignas, por sua vez, foram classificadas segundo o grau de diferenciação em: bem diferenciado, moderadamente diferenciado, pouco diferenciado ou indiferenciado 9 .

Foram consideradas lesões sincrônicas a presença de mais de uma lesão ao exame ou nova lesão diagnosticada até dois anos do último diagnóstico endoscópico de lesão polipoide, e as lesões metacrônicas, como o aparecimento de nova lesão após dois anos do último diagnóstico de lesão polipoide.

A presente pesquisa foi aprovada pelo Comitê de Ética em Pesquisa da Universidade Federal de Sergipe sob o número 0145.0.107.000-07. A pesquisa faz uso exclusivamente dos dados contidos nos prontuários dos pacientes e, para cumprimento dos preceitos éticos, foi mantido o sigilo sobre a identidade desses pacientes.

Após a coleta, os dados foram analisados com a elaboração de gráficos e tabelas em planilhas do programa Microsoft Excel 2007. Os resultados foram confrontados com dados encontrados na literatura durante todo o decorrer do estudo por meio de revisão bibliográfica. Para análise estatística, utilizou-se o programa Epi-Info Versão 3.4.3, 2007. Foi adotado nível de significância de $5 \%(\mathrm{p} \leq 0,05)$, sendo aplicado o teste do qui-quadrado não corrigido com variação 
bicaudal para duas tabelas 2 × 2 , com o objetivo de analisar as associações entre as variáveis estudadas: tipo histológico e grau de displasia; tamanho do adenoma e grau de displasia.

\section{RESULTADOS}

Foram analisados 6.919 exames endoscópicos referentes a 2.126 retossigmoidoscopias e a 4.793 vídeocolonoscopias realizadas no Serviço de Coloproctologia do Hospital Universitário de Sergipe e no Torres Centro Médico, no período de janeiro de 2002 a dezembro de 2007, realizados por oito endoscopistas que compõem o corpo clínico do serviço estudado, sendo $57 \%$ destes exames realizados por um único examinador.

Dos 6.919 exames analisados, foram encontrados 1.031 pólipos e lesões plano-elevadas colorretais em 935 exames $(13,51 \%)$. Setecentos e noventa e um deles $(84,60 \%)$ foram vistos à colonoscopia e 144 $(15,40 \%)$ vistos à retossigmoidoscopia, perfazendo um total de 826 pacientes.

Dos 826 pacientes submetidos a 935 exames, $753(91,20 \%)$ deles se submeteram a um exame, 50 $(6,10 \%)$ pacientes a dois exames, $14(1,70 \%)$ a três exames, $6(0,70 \%)$ a quatro exames, $2(0,20 \%)$ a cinco exames e $1(0,10 \%)$ paciente foi submetido a sete exames, com média de 1,13 exames por paciente.

Dos 826 pacientes examinados, 380 pacientes (46\%) eram do gênero masculino e 446 (54\%) do gênero feminino (Gráfico 1). A média de idade foi de $53,64 \pm 16,34$ anos, com extremos de 3 e 96 anos. Em relação à sua distribuição, $68(8,20 \%)$ tinham até 30 anos ao exame, $66(8,00 \%)$ estavam na faixa etária de 31-40 anos, $192(23,20 \%)$ entre 41-50 anos, 206 $(24,90 \%)$ entre 51-60 anos, 171 (20,70\%) entre 61-70 anos, 90 (10,90\%) entre 71-80 anos, $26(3,10 \%)$ apresentavam 80 anos ou mais e a idade de $7(0,80 \%)$ deles não foi referida. Quanto à procedência, $552(66,80 \%)$ eram provenientes de Aracaju (Capital), 243 (29,40\%) do interior do Estado, 11 (1,30\%) de estados vizinhos (Alagoas e Bahia) e 20 deles (2,40\%) não tinham essa variável referida no exame (Gráfico 2).

Dos 935 exames selecionados, $699(74,80 \%)$ apresentavam lesão polipoide única, 154 (16,50\%) apresentavam duas lesões, 55 (5,90\%) três lesões, $15(1,60 \%)$ quatro lesões, $8(0,90 \%)$ cinco lesões, 4 $(0,40 \%)$ seis lesões (Tabela 1$)$.
As indicações para a realização destes exames foram: sangramento retal em 117 casos $(9,80 \%)$, dor anorretal em $33(2,80 \%)$, dor abdominal em $110(9,20 \%)$, diarreia em $41(3,40 \%)$, constipação intestinal em 62 (5,20\%), alteração do hábito intestinal em 38 (3,10\%), seguimento de polipectomia em $89(7,40 \%)$, seguimento de outras doenças colônicas (principalmente a doença diverticular dos cólons e as doenças inflamatórias intestinais) em 58 casos $(4,90 \%)$, pólipo visto à retossigmoidoscopia em 17 (1,40\%), doença hemorroidária em $73(6,10 \%)$, síndrome fissurária em $10(0,8 \%)$, sangramento oculto nas fezes em $8(0,7 \%)$, história familiar de neoplasia de cólon em $17(1,40 \%)$, controle de

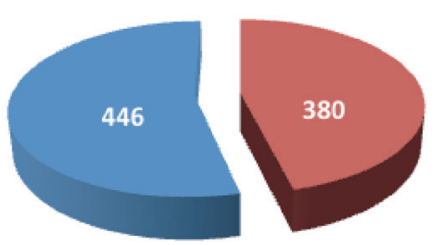

homens

mulheres

Gráfico 1 - Distribuição dos pacientes por gênero

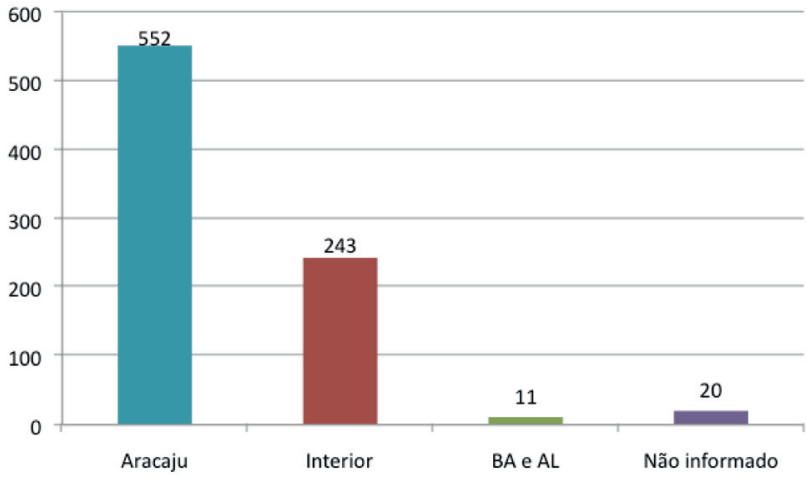

Gráfico 2 - Distribuição dos pacientes quanto à procedência

Tabela 1 - Número de pólipos encontrados por exame

\begin{tabular}{lcc}
\hline $\mathbf{N}^{\mathbf{0}}$ de lesões & $\mathbf{N}^{\mathbf{0}}$ de exames & Percentual \\
\hline 1 & 699 & 74,8 \\
2 & 154 & 16,5 \\
3 & 55 & 5,9 \\
4 & 15 & 1,6 \\
5 & 8 & 0,9 \\
6 & 4 & 0,4 \\
Total & 935 & 100,0 \\
\hline
\end{tabular}


neoplasia de cólon em $8(0,70 \%)$ e outras indicações em 92 casos (7,80\%). Em 422 exames (35,30\%) não havia informação sobre a sua indicação (Tabela 2).

Os diagnósticos associados a lesões polipoides foram: doença hemorroidária em 268 casos $(22,00 \%)$, doença diverticular dos cólons em 244 (20,10\%), coli-

Tabela 2 - Indicação do exame endoscópico

\begin{tabular}{lcc}
\hline Indicações & Número & Porcentagem \\
\hline Sangramento retal & 117 & 9,8 \\
Dor anorretal & 33 & 2,8 \\
Dor abdominal & 110 & 9,2 \\
Diarreia & 41 & 3,4 \\
Constipação & 62 & 5,2 \\
Controle pós-polipectomia & 89 & 7,4 \\
Controle de outras doenças & 58 & 4,9 \\
colônicas & & \\
Pólipo visto à & 17 & 1,4 \\
retossigmoidoscopia & & \\
Doença hemorroidária & 73 & 6,1 \\
História familiar de & 17 & 1,4 \\
neoplasia de cólon & & \\
Alteração do hábito & 38 & 3,1 \\
intestinal & & \\
Sangramento oculto nas & 8 & 0,7 \\
fezes & & \\
Controle de neoplasia de & 8 & 0,7 \\
cólon & & \\
Síndrome fissurária & 10 & 0,8 \\
Não informa & 422 & 35,3 \\
Outras & 92 & 7,8 \\
Total & 1.195 & 100 \\
\hline
\end{tabular}

Tabela 3 - Classificação das lesões polipoides estudadas

\begin{tabular}{lcc}
\hline Tipo & $\begin{array}{c}\mathbf{N}^{0} \text { de lesões } \\
\text { polipoides }\end{array}$ & $\begin{array}{c}\text { Frequência } \\
\text { (\%) }\end{array}$ \\
\hline Adenoma tubular & 383 & 29,6 \\
Adenoma túbulo-viloso & 43 & 3,3 \\
Adenoma viloso & 21 & 1,6 \\
Não adenomatoso & 508 & 39,2 \\
Misto & 25 & 1,9 \\
Lesão maligna & 9 & 0,7 \\
Mucosa sem alteração & 42 & 3,2 \\
histológica & & \\
Total & 1.031 & 100 \\
\hline
\end{tabular}

te em $62(5,10 \%)$, síndrome fissurária em $29(2,40 \%)$, papilite e/ou papilas hipertróficas em 25 (2,10\%), plicomas em 23 (1,90\%), neoplasia de cólon em 17 $(1,40 \%)$, retocolite ulcerativa idiopática (RCUI) em $14(1,2 \%)$, proctite em $14(1,20 \%)$, lipoma em 13 $(1,10 \%)$ e outros diagnósticos em $119(9,80 \%)$ casos, tais quais angiectasias, aderências, ileíte, tiflite, melanose coli, entre outros. Em 388 (31,90\%) exames analisados não havia outro diagnóstico associado.

As 1.031 lesões polipoides analisadas apresentaram a seguinte distribuição topográfica: $316(30,60 \%)$ lesões no cólon sigmoide, $287(27,80 \%)$ no reto, 144 $(14,00 \%)$ no cólon transverso, $80(7,80 \%)$ no ceco, $79(7,70 \%)$ no cólon descendente, 78 (7,60\%) no cólon ascendente, $18(1,70 \%)$ no ângulo hepático, 10 $(1,00 \%)$ no retossigmoide, $9(0,90 \%)$ no ângulo esplênico. Em 10 casos (1\%) não havia informação.

Quanto ao tamanho, 864 lesões polipoides tinham o tamanho descrito no exame. Quinhentos e sessenta e nove $(65,90 \%)$ tinham até $10 \mathrm{~mm}$ de diâmetro, $261(30,20 \%)$ entre 10 e $20 \mathrm{~mm}, 34(3,90 \%)$ tinham $21 \mathrm{~mm}$ ou mais.

Das 1.031 lesões, 767 lesões tinham o aspecto morfológico descrito. Destes, 405 (52,80\%) eram sésseis, $214(27,90 \%)$ pediculados e $148(19,30 \%)$ eram plano-elevados.

Das 1.031 lesões polipoides colorretais analisadas, 447 (43,35\%) eram adenomas, 508 (39,20\%) eram lesões não-adenomatosas, 25 eram lesões polipoides mistas (Tabela 3). Foram diagnosticadas, ainda, 9 lesões malignas, 7 (77,80\%) adenocarcinomas, $1(11,10 \%)$ tumor carcinoide e $1(11,10 \%)$ tumor gastrointestinal estromal (GIST).

Das lesões não-adenomatosas, 316 (62,20\%) eram lesões polipoides hiperplásicas, $163(32,10 \%)$

Tabela 4 - Tipo histológico das lesões nãoadenomatosas analisadas

\begin{tabular}{lcc}
\hline Não-adenomatoso & Frequência & Percentual \\
\hline Hiperplásico & 316 & 62,2 \\
Inflamatório & 163 & 32,1 \\
Hamartoma & 11 & 2,2 \\
Lipoma & 5 & 1,0 \\
Hiperplasia linfoide & 7 & 1,4 \\
Granuloma parasitário & 5 & 1,0 \\
Leiomioma & 1 & 0,2 \\
Total & 508 & 100,0 \\
\hline
\end{tabular}


lesões polipoides inflamatórias, 5 (1,00\%) lipomas, $11(2,20 \%)$ hamartomas, $7(1,40 \%)$ hiperplasias linfoides, $1(0,20 \%)$ leiomioma submucoso e $5(1,00 \%)$ granulomas parasitários (Tabela 4).

Das 447 lesões adenomatosas, 383 (85,70\%) eram adenomas tubulares, $43(9,60 \%)$ adenomas túbulo-vilosos e 21 (4,70\%) adenomas vilosos.

Quanto ao grau de displasia, $373(83,40 \%)$ eram adenomas de baixo grau e $74(16,60 \%)$ adenomas de alto grau. Dos 383 adenomas tubulares, 339 (88,50\%) eram considerados de baixo grau e $44(11,50 \%)$ de alto grau. Para os 43 adenomas túbulo-vilosos, 25 $(58,10 \%)$ eram de baixo grau e $18(41,90 \%)$ de alto grau. E dos 21 adenomas vilosos, 9 (42,90\%) eram de baixo grau e $12(57,10 \%)$ de alto grau (Tabela 5$)$.

Correlacionando-se o tamanho do adenoma e o grau de displasia, dos 284 adenomas menores ou iguais a $10 \mathrm{~mm}, 254(89,40 \%)$ eram de baixo grau e $30(10,60 \%)$ de alto grau. Dos 110 adenomas com mais de $10 \mathrm{~mm}, 80(72,70 \%)$ eram de baixo grau e 30 $(27,30 \%)$ de alto grau (Tabela 6).

Das 1.031 lesões polipoides observadas, 194 $(20,90 \%)$ lesões eram plano-elevadas, das quais 6 $(3,10 \%)$ eram lesões mistas, $31(6,95 \%)$ eram adenomatosas, com $25(80,60 \%)$ apresentando displasia de baixo grau e $6(12,90 \%)$ displasia de alto grau.

Dos $7(77,80 \%)$ adenocarcinomas diagnosticados ao exame histopatológico, 3 tinham focos de adenoma, todos com histopatológico de adenoma túbulo- viloso. Quanto ao grau de diferenciação, 2 (28,60\%) eram bem diferenciados, $2(28,60 \%)$ moderadamente diferenciados e o grau de diferenciação não foi referido em $3(42,90 \%)$ deles.

Dos 826 pacientes estudados, 194 (23,48\%) apresentaram lesões sincrônicas. Destes, 174 pacientes apresentaram mais de uma lesão no mesmo exame e 50 apresentaram diagnóstico de nova lesão até 2 anos após a última lesão diagnosticada. Nos 73 pacientes submetidos a mais de um exame, lesões metacrônicas foram observadas em $22(30,10 \%)$ casos.

\section{DISCUSSÃO}

O estudo dos pólipos e lesões plano-elevadas colorretais tem um significado clínico importante na diminuição da incidência e diagnóstico precoce do câncer colorretal (CCR). Isso se deve ao potencial de malignização dos adenomas, lesões pré-malignas bem definidas que antecedem em 10 a 15 anos o câncer colorretal $^{3,4}$. Programas de rastreamento destas lesões permitem sua detecção e remoção, interrompendo a progressão adenoma-câncer e prevenindo o câncer, além de possibilitar a detecção do câncer em estágio inicial, podendo elevar a taxa de sobrevida em 5 anos a $90 \%$ quando adequadamente tratado ${ }^{10}$.

Winawer et al. mostraram, através de seus trabalhos, que os programas de rastreamento endoscópico para pacientes de risco aumentado, ou seja, que

Tabela 5 - Relação entre o tipo histológico do adenoma e o grau de displasia

\begin{tabular}{lccccc}
\hline \multirow{2}{*}{ Tipo do adenoma } & \multicolumn{2}{c}{ Grau de displasia $\mathbf{n}^{\mathbf{0}}$} & \multicolumn{2}{c}{ Grau de displasia \% } & \multirow{2}{*}{ Valor de p* } \\
\cline { 2 - 5 } & Baixo & Alto & Baixo & Alto & \\
\hline Tubular & 339 & 44 & 88,5 & 11,5 & \\
Túbulo-viloso & 25 & 18 & 58,1 & 41,9 & $<0,001$ \\
Viloso & 9 & 12 & 42,9 & 57,1 & \\
\hline
\end{tabular}

*Teste qui-quadrado: tipo histológico versus grau de displasia $(p<0,001)$.

Tabela 6 - Relação entre tamanho das lesões adenomatosas e grau de displasia

\begin{tabular}{lccccc}
\hline \multirow{2}{*}{ Tamanho do adenoma } & \multicolumn{2}{c}{ Grau de displasia $\mathbf{n}^{\mathbf{0}}$} & \multicolumn{2}{c}{ Grau de displasia \% } & \multirow{2}{*}{ Valor de $\mathbf{p}^{*}$} \\
\cline { 2 - 4 } & Baixo & Alto & Baixo & Alto & \\
\hline $0-10 \mathrm{~mm}$ & 254 & 30 & 89,4 & 10,6 & $<0,001$ \\
$11 \mathrm{~mm}$ ou mais & 80 & 30 & 72,7 & 27,3 & $<0,001$ \\
\hline
\end{tabular}

*Teste qui-quadrado: tamanho do adenoma versus grau de displasia $(p<0,001)$. 
tenham história familiar positiva ou história pessoal de pólipos adenomatosos, câncer colorretal e doença inflamatória intestinal, são importantes para diminuir a mortalidade por CCR. Em seu estudo, a polipectomia endoscópica das lesões adenomatosas precursoras reduziu a mortalidade por CCR para a população rastreada por colonoscopia em até $90 \%$, o que mostra a sua importância no controle da progressão para o câncer ${ }^{11}$.

Diversos trabalhos mostraram que as lesões polipoides incidem com relativa frequência como achados endoscópicos, principalmente por colonoscopia. Segundo Winawer, as taxas observadas estão em torno de $20 \%{ }^{12}$. Em nosso trabalho, foi observada uma incidência de $13,51 \%$.

A média de idade dos pacientes avaliados foi de $53,64 \pm 16,34$ anos, com extremos de 3 e 96 anos. Dos 826 pacientes estudados, $467(56,50 \%)$ estavam na faixa etária de 51 a 80 anos, sendo estas três décadas as mais acometidas, à semelhança do relatado por outros autores ${ }^{13-16}$.

A incidência por gênero, que neste estudo mostrou valores de $46 \%$ no gênero masculino e $54 \%$ no gênero feminino, apresentou uma frequência relativamente maior da população feminina, diferentemente do observado em dados da literatura, que não mostraram diferença significativa entre homens e mulheres ${ }^{15,16}$. No entanto, esse dado foi condizente com os dados do Instituto Nacional do Câncer (INCA) em relação à incidência por gênero do câncer colorretal, em que o número de casos novos estimados para o Brasil no ano de 2008 é de 12.490 casos em homens e 14.500 em mulheres. Estes valores correspondem a um risco estimado de 13 casos novos a cada 100 mil homens e 15 a cada 100 mil mulheres, mostrando uma maior prevalência nas mulheres ${ }^{8}$.

As indicações mais frequentes para a realização do exame endoscópico foram: sangramento retal, dor abdominal, seguimento de polipectomia, constipação intestinal, seguimento de outras doenças colônicas, diarreia e história familiar de neoplasia de cólon, indicações semelhantes às relatadas nos estudos pesquisados ${ }^{12,17}$. A maioria dessas indicações são sintomas relacionados à prevenção do câncer.

A importância da colonoscopia vai além do diagnóstico dos pólipos, objeto do nosso estudo. Dos exames realizados, cujos pacientes eram portadores de pólipos, foram feitos outros diagnósticos associados de patologias como a doença hemorroidária (22\%), doença diverticular dos cólons $(20,10 \%)$, colites $(5,10 \%)$, síndrome fissurária $(2,40 \%)$, RCUI $(1,20 \%)$, entre outros.

A maior prevalência dos pólipos foi no sigmoide $(30,60 \%)$, seguido do reto $(27,80 \%)$. Quando somamos as duas distribuições topográficas, observamos que $58,40 \%$ são localizados no reto e sigmoide, também correspondendo a maior incidência do câncer colorretal. Estas lesões também estariam ao alcance da retossigmoidoscopia flexível. No entanto, observamos que lesões do ceco até o cólon transverso, não acessíveis por retossigmoidoscopia, teriam passado despercebidas nos programas de prevenção do câncer colorretal que advogam a utilização desse método. Nas publicações mais recentes, tem aumentado o diagnóstico de lesões proximais, apontando para uma mudança do comportamento do câncer colorretal e revelando uma melhor acurácia com a utilização da colonoscopia. No nosso estudo, foram encontrados $31,10 \%$ das lesões no cólon direito. Num estudo analisando a distribuição dos tumores colorretais em 1.694 neoplasias durante os períodos de 1960-1969, 1970-1979 e 1980-1984, foi observado um decréscimo final de $15,80 \%$ para neoplasias do reto e um aumento de 10,20\% para as do cólon direito, evidenciando essa mudança de comportamento do câncer colorretal $^{18}$.

Em relação ao aspecto morfológico, das 767 lesões descritas, 405 (52,80\%) eram sésseis, 214 $(27,90 \%)$ pediculadas e $148(19,30 \%)$ eram planoelevadas. Dados da literatura apontam uma maior prevalência das lesões sésseis ${ }^{16}$. O conceito inicial de prevenção do câncer colorretal era centrado no diagnóstico e tratamento de lesões polipoides maiores e de diagnóstico mais fácil. Porém, estudos japoneses têm chamado a atenção para as lesões planas e planoelevadas, de coloração muito semelhante à mucosa adjacente, e que podem passar despercebidas ao examinador menos atento. Na nossa casuística, 19,30\% dessas lesões foram diagnosticadas. Admite-se que, apesar de endoscopistas experientes alcançarem o ceco muito rapidamente, a colonoscopia é um exame a ser realizado principalmente durante a retirada do aparelho, momento em que se diagnostica um maior número de lesões. Porém, as lesões identificadas du- 
rante a introdução do aparelho devem ser prontamente ressecadas ou marcadas para que não se percam quando da retirada do mesmo.

Barclay et al. ${ }^{19}$ compararam a incidência de pólipos colorretais entre examinadores que levavam menos e mais de seis minutos para realizar o exame colonoscópico. Os examinadores que levaram menos de 6 minutos diagnosticaram pólipos em $11,80 \%$ das colonoscopias, enquanto os que levaram mais de $6 \mathrm{mi}-$ nutos encontraram pólipos em $28,30 \%$.

Diversos trabalhos demonstram que os pólipos mais comumente encontrados no intestino grosso são os hiperplásicos e os adenomatosos. Como visto, os adenomas tem o tipo histológico considerado mais importante do ponto de vista diagnóstico devido ao seu potencial de transformação maligna, e podem representar até metade a dois terços do total das lesões polipoides ${ }^{20}$. Dos vários trabalhos consultados, foi visto que as lesões adenomatosas podem tanto ser mais ${ }^{21-24}$ como menos frequentes ${ }^{10,25,26}$, ou até mostrar incidência semelhante aos hiperplásicos ${ }^{26,27}$. Em nossa casuística, os adenomas foram o tipo histológico mais frequente, com 447 (43,35\%) lesões encontradas, seguido pelos hiperplásicos com 316 (30,65\%) lesões polipoides.

Os pólipos hiperplásicos são considerados lesões sem nenhum potencial de transformação maligna, conceito que tem sido questionado com o reconhecimento do adenoma serrilhado ${ }^{28,29}$. É considerada uma lesão intermediária caracterizada pela associação de tecido hiperplásico e adenomatoso, com evidências de que podem estar associados à neoplasia colorretal ${ }^{30}$. Neste estudo, foram encontrados 25 casos de adenoma serrilhado. Isso reforça a importância, relatada por alguns autores, da remoção dessas lesões e seu estudo histopatológico quando não é possível distinguir pólipos hiperplásicos de possíveis adenomas durante o exame endoscópico ${ }^{28,29}$. Estudos têm sido realizados para distinguir endoscopicamente as referidas lesões, tais como cromoendoscopia com ou sem colonoscopia de alta resolução e alta magnificação.

Em relação às 447 lesões adenomatosas analisadas, $383(85,70 \%)$ eram adenomas tubulares, 43 $(9,60 \%)$ adenomas túbulo-vilosos e $21(4,70 \%)$ adenomas vilosos. Os artigos revisados mostraram que o tipo tubular é o mais frequentemente encontrado, variando de 39 a 80,50\%. Menos comuns, o túbulo-vilo- so variam entre 3 e $55,70 \%$, e o tipo viloso entre 4,30 e $17 \%^{14,31}$. Vimos, portanto, uma semelhança dos nossos dados com os da literatura, embora o percentual de adenomas tubulares por nós diagnosticados esteja acima do relatado.

Dados da World Health Organization Classification of Tumours, visando à padronização dos resultados dos exames anatomopatológicos e, consequentemente, das publicações, orienta desde 2000 que as displasias sejam classificadas em duas categorias: baixo e alto grau. Na revisão dos laudos anatomopatológicos, ainda encontramos um número significativo de relato de displasias classificadas como leve, moderada e intensa ${ }^{9}$. Neste estudo, foram observados $373(83,40 \%)$ adenomas de baixo grau e $74(16,60 \%)$ adenomas de alto grau. Segundo alguns autores, a displasia de alto grau está presente em 2 a $5 \%$ dos adenomas, portanto, bem inferior aos nossos dados ${ }^{10,24}$. Analisando separadamente os adenomas de acordo com o tipo histológico, haja vista o maior potencial de transformação maligna imputado aos adenomas vilosos, observamos que dos 383 adenomas tubulares, $339(88,50 \%)$ eram considerados de baixo grau e $44(11,50 \%)$ de alto grau. Para os 43 adenomas túbulo-vilosos, $25(58,10 \%)$ eram de baixo grau e $18(41,90 \%)$ de alto grau. Dentre os 21 adenomas vilosos, $9(42,90 \%)$ eram de baixo grau e $12(57,10 \%)$ de alto grau, sendo esta comparação, de tipo histológico e grau de displasia, estatisticamente significante $(\mathrm{p}<0,001)$. Estes dados demonstram que a presença do componente viloso aumenta o potencial de transformação maligna, confirmada pela maior incidência de displasia de alto grau nos grupos túbulo-viloso e viloso, dados relatados pela maioria dos autores ${ }^{32-36}$.

Morson et al., em 1976, já estabelecia que a lesão precursora do CCR é o pólipo adenomatoso e que seu potencial de transformação maligna é proporcional ao tamanho, ao componente viloso e ao grau de displasia. Schmiegel et al. ${ }^{37}$ também concluíram que os fatores associados com a maior taxa de desenvolvimento de câncer são diâmetro maior que $10 \mathrm{~mm}$, presença de componente viloso ou atipias acentuadas.

Correlacionado o tamanho dos adenomas e o grau de displasia, dos 284 adenomas menores ou iguais a $10 \mathrm{~mm}, 254(89,40 \%)$ eram de baixo grau e $30(10,60 \%)$ de alto grau. Dos 110 adenomas maio- 
res que $10 \mathrm{~mm}, 80(72,70 \%)$ eram de baixo grau e 30 $(27,30 \%)$ de alto grau. Essa comparação mostrou que o tamanho também influencia no potencial de malignização, também confirmado pela maior incidência de displasia de alto grau que ocorreu em até $10,60 \%$ das lesões menores ou iguais a $10 \mathrm{~mm}$, e em $27,30 \%$ das maiores que $10 \mathrm{~mm}$, sendo esta comparação estatisticamente significante $(\mathrm{p}<0,001)$. $\mathrm{O}$ mesmo foi observado em outros estudos nos quais o aumento do tamanho dos pólipos também aumenta a incidência de displasia de alto grau ${ }^{16,34-36}$.

Dos pólipos diagnosticados, 7 (2,01\%) foram considerados malignas ao exame histopatológico. Seitz et al. ${ }^{38}$ descreveram uma variação na taxa de transformação maligna dos adenomas entre 2 e $9,4 \%$, taxa que foi descrita por Bond ${ }^{34}$ entre 4 e $7 \%$ e por Schmiegel et al. ${ }^{37}$ em 5\%. Foram diagnosticados, ainda, um tumor carcinoide e outro tumor gastrintestinal estromal (GIST) ${ }^{16}$. Dos sete adenocarcinomas observados, três deles tinham focos de adenoma na lesão, todos com resultado histopatológico de adenoma túbulo-viloso. Todos os 7 adenocarcinomas eram pólipos com tamanho variando de 10 a $75 \mathrm{~mm}$. Quanto ao grau de diferenciação, 2 $(28,60 \%)$ eram bem diferenciados, $2(28,60 \%)$ moderadamente diferenciados e o grau de diferenciação não foi referido em 3 deles $(42,90 \%)$. Tem-se atribuído às lesões plano-elevadas uma maior incidência de displasia de alto grau. Quando comparamos as lesões protrusas com as plano-elevadas, observou-se que aquelas tinham uma incidência de displasia de alto grau de $7,61 \%$, menores que os $12,90 \%$ das lesões plano-elevadas. No entanto, não encontramos nenhuma lesão maligna dentre as lesões plano-elevadas e nenhuma lesão plano-elevada menor que $5 \mathrm{~mm}$ com displasia de alto grau.

Pólipos sincrônicos são relatados entre 18,90 e $37,40 \%$ dos pacientes ${ }^{16}$. Dos 826 pacientes estudados, $194(23,48 \%)$ apresentaram lesões sincrônicas. Destes, 174 apresentaram mais de uma lesão no mesmo exame e 50 apresentaram diagnóstico de nova lesão até 2 anos após a última lesão diagnosticada. Este número foi superior ao total porque havia pacientes que se enquadravam nos dois critérios: mais de uma lesão ao exame e aparecimento de nova lesão até dois anos após a última lesão diagnosticada. Entre os 73 pacientes submetidos a mais de um exame, lesões metacrônicas, ou seja, após dois anos do último diagnóstico de lesão polipoide, foram observadas em $22(30,10 \%)$ pacientes.

Segundo o National Polyp Study ${ }^{39}$, após a ressecção dos adenomas, há um risco de ocorrência de novas lesões estimado entre 32 a 41,7\%, taxa semelhante ao encontrado em nosso estudo, o que mostra a importância do seguimento destes pacientes após a polipectomia.

\section{CONCLUSÃO}

Foram diagnosticados pólipos em 13,51\% dos pacientes submetidos a colonoscopia e retossigmoidoscopia. Os adenomas foram os mais frequentes, e são importantes para carcinogênese colorretal.

Topograficamente, foram mais frequentes no reto e sigmoide, correspondendo à localização preferencial dos cânceres colorretais e acessíveis por retossigmoidoscopia. No entanto, $31,10 \%$ das lesões foram encontradas no cólon direito, portanto não acessíveis por retossigmoidoscopia, o que mostra a importância da utilização da colonoscopia como método diagnóstico dessas lesões.

Foi verificado que a presença do componente viloso aumenta o potencial de transformação maligna, o que foi confirmado pela maior incidência de displasia de alto grau nos adenomas túbulo-vilosos e vilosos. Houve, também, maior incidência de displasia de alto grau nos adenomas maiores, ratificando o maior risco de malignização com o aumento do tamanho da lesão.

Em sete pacientes, foram diagnosticados pólipos adenomatosos já malignos, além de um GIST e um tumor carcinoide.

Nos pacientes submetidos a mais de um exame, foi visto um alto índice de ocorrência de novas lesões, mostrando a importância do seguimento colonoscópico de pacientes portadores de adenomas colorretais.

Conclui-se que a colonoscopia e a polipectomia são eficazes no diagnóstico e prevenção do câncer colorretal. 
ABSTRACT: Introduction: Colorectal polyps and flat injuries are important for the prevention of colorectal cancer due to the malignancy of adenomas. Objective: To describe the demographic profile of patients with endoscopic diagnosis of colorectal polyps and/or flat injuries at University Hospital of Universidade Federal de Sergipe and Torres Medical Center. Methods: A total of 6,919 records were evaluated from January 2002 to December 2007 with respect to the following variables: age, gender, origin, indication; injury evaluation os number, size, morphology, histology, dysplasia degree, topography; synchronics and metachronics injuries and associated diagnoses. Results: 1,031(13.51\%) colorectal polyps and flat injuries were found in 935 tests, accounting for 826 patients, $\mathbf{4 6 \%}$ males and $\mathbf{5 4 \%}$ females. The age ranged from 3 to 96 years with average of 53.64. The most frequent topographic distribution of polypoid injuries were rectum and sigmoid (58.40\%). The injuries were sessile in $52.80 \%$ of the cases, pedicle in $27.90 \%$, and flat injuries in $\mathbf{1 9 . 3 0} \%$. There were synchronical injuries in $\mathbf{2 3 . 4 8 \%}$ of patients and metachronic in 22 patients. Histologically, $\mathbf{4 3 . 3 6 \%}$ were adenomas, being $85.70 \%$ tubular, $9.60 \%$ tubulovillous and $4.70 \%$ villous; hyperplastic polyps were found in $30.64 \%$ of the cases, inflammatory in $\mathbf{1 5 . 8 0} \%$ and $\mathbf{1 0 . 2 0} \%$ presented other histological types. The adenomas showed low-grade dysplasia in $\mathbf{8 3 . 4 0 \%}$ and high degree in $16.60 \%$ of the patients. Seven were adenoma-carcinomas, one was carcinoid and one was gastrointestinal stromal tumor. Conclusions: The colonoscopy and polypectomy are important in diagnosis and prevention of colorectal cancer.

Key words: Adenoma. Polyps. Colonoscopy.

\section{REFERÊNCIAS}

1. Galvão Alves, J. Terapêutica em gastroenterologia. Guanabara Koogan; 2005.

2. Bogliolo. Patologia. $6^{\mathrm{a}}$ ed. Guanabara Koogan; 2000.

3. Stryker SJ, Wolff BG, Culp CE, Libbe SD, Ilstrup DM, MacCarty RL. Natural history of untreated colonic polyps. Gastroenterology. 1987;93(5):1009-13.

4. Durado D. Brooks, Sidney J. Winawer, Douglas K. Rex, Ann G. Zauber, Charles j. Kahi, et al. Colonoscopy Surveillance After Polypectomy and Colorectal Cancer Resection Consensus Guidelines from the U.S. Multi-Society Task Force on Colorectal Cancer and the American Cancer Society. 2008;77(7):995-1002, 1003-1004. Copyright C 2008 American Academy of Family Physicians.

5. Kudo S. Endoscopic mucosal resection of flat and depressed types of early colorectal cancer. Endoscopy 1993;25(7): 455-61.

6. Ansher AF, Lewis JH, Fleischer DE, Cattau EL Jr, Collen MJ, O'Kieffe DA, et al. Hyperplastic colonic polyps as a marker adenomatous colonic polyps. American Journal of Gastroenterology 1989;84(2):113-7.

7. Patel K, Hoffman NE. The anatomical distribution of colorectal polyps at colonoscopy. J Clin Gastroenterol 2001;33(3):222-5.

8. Instituto Nacional de Câncer [homepage na internet]. Brasil: Ministério da Saúde c1996-2007 [citado 2008 Aug 30]. Disponível em: http://www.inca.gov.br/

9. Hamilton SR, Aaltonen LA, editor. World Health Organization Classification of Tumours. Pathology and Genetics of Tumours of the Digestive System. IARC Press: Lyon; 2000.

10. Fenoglio-Preiser CM, Hutter RVP. Colorectal Polyps: Pathologic Diagnosis and Clinical Significance. CA Cancer J Clin 1985;35:322-44.

11. Winawer SJ, Zauber AG, Brien MJ, Gottlieb LS, Sternberg SS, Bond JH, Waye JD, May Nah Ho, et al. Randomized comparison of surveillance intervals after colonoscopic removal of newly diagnosed adenomatous polyps. N Engl J Med 1993;328(13):901-6.

12. Santos JM dos, Felício F, Oliveira JCC de, Silva CA, Schneider EJ. Tratamento endoscópico dos pólipos colorretais. Arq Cat Med. 1996;25(3):230-5.

13. Collett JA, Olynyk JK, Platell CF. Flexible sigmoidoscopy screening for colorectal cancer in average-risk people: update of a community-based project. Med J Aust 2000;173(9):463-6.

14. Kapsoritakis AN, Potamianos SP, Koukourakis MI, Tzardi M, Mouzas IA, Roussomoustakaki M, et al. Diminutive polyps of large bowel should be an early target for endoscopic treatment. Dig Liver Dis 2002;34(2):137-40.

15. Almeida MG, Baraviera AC, Malheiros APR, Bellandi DM, Cury RM, Milman MHSA, et al. Polipectomias endoscópicas - estudo histopatológico e complicações. Rev bras Coloproct 2003;23(2):100-4.

16. Manzione CR, Nadal SR, Nadal MA, Melo SVM. Análise Morfológica e Histológica de Pólipos Colorretais Submetidos à Ressecção Endoscópica. Rev Bras Coloproct 2004;24(2):119-25.

17. Church JM. Experience in the endoscopic management of large colonic polyps. ANZ J Surg. 2003;73(12):988-95.

18. Gharemani GG, Dowlatshahi K. Colorectal carcinomas: diagnostic implications of their changing frequency and anatomic distribution. World J Surg 1989;13(3):321-5.

19. Barclay RL, Vicari JJ, Doughty AS, Johanson JF, Greenlaw RL. Colonoscopic withdrawal times and adenoma detection during screening colonoscopy. N Engl J Med. 2006;355(24):2533-41.

20. Levin B, Lieberman DA, McFarland B, Smith RA, Brooks D, Andrews KS, Dash C, Giardiello FM, Glick S, Levin TR, PickhardtP, Rex DK, Thorson A, Winawer SJ; American Cancer Society Colorectal Cancer Advisory Group; US Multi-Society Task Force; American College of Radiology Colon Cancer Committee. Screening and surveillance for the early detection of colorectal cancer and adenomatous polyps, 2008: a joint 
guideline from the American Cancer Society, the US MultiSociety Task Force on Colorectal Cancer, and the American College of Radiology. CA Cancer J Clin 2008;58(3):130-60.

21. Zheng S, Liu XY, Ding KF, Wang LB, Qiu PL, Ding XF, et al. Reduction of the incidence and mortality of rectal cancer by polypectomy: a prospective cohort study in Haining County. World J Gastroenterol 2002;8(3):488-92.

22. Morimoto LM, Newcomb PA, Ulrich CM, Bostick RM, Lais CJ, Potter JD. Risk factors for hyperplastic and adenomatous polyps: evidence for malignant potential? Cancer Epidemiol Biomarkers Prev 2002;11(10 Pt 1):1012-8.

23. Patel K, Hoffman NE. The anatomical distribution of colorectal polyps at colonoscopy. J Clin Gastroenterol 2001;33(3):222-5.

24. Webb WA, McDaniel L, Jones L. Experience with 1000 colonoscopic polypectomies. Ann Surg 1985;201(5):626-32.

25. Williams AR, Balasooriya BA, Day DW. Polyps and cancer of the large bowel: a: necropsy study in Liverpool. Gut 1982;23(10):835-42.

26. Liljegren A, Lindblom A, Rotstein S, Nilsson B, Rubio C, Jaramillo E. Prevalence and incidence of hyperplastic polyps and adenomas in familial colorectal cancer: correlation between the two types of colon polyps. Gut 2003;52(8):1140-7.

27. Imperiale TF, Wagner DR, Lin CY, Larkin GN, Rogge JD, Ransohoff DF. Results of screening colonoscopy among persons 40 to 49 years of age. N Engl J Med 2002;346(23):1781-5.

28. Huang CS, O'Brien M J, Yang S, Farraye FA. Hyperplastic polyps, serrated adenomas, and the serrated polyp neoplasia pathway. Am J Gastroenterol. 2004;99(11):2242-55.

29. Higuchi T, Jass JR. My approach to serrated polyps of the colorectum. J Clin Pathol. 2004;57(7):682-6.

30. Cross SS. Kappa statistics as indicators of quality assurance in histopathology and cytopathology. J Clin Pathol 1996;49(7):597-9.
31. Rubio CA. Colorectal adenomas: time for reappraisal. Pathol Res Pract 2002;198(9):615-20.

32. Khan A, Shrier I, Gordon PH. Do distal adenomas mandate total colonoscopy? Surg Endosc 2003;17(6):886-90.

33. Lieberman DA, Weiss DG, Bond JH, Ahnen DJ, Garewal $\mathrm{H}$, Chejfec G. Use of colonoscopy to screen asymptomatic adults for colorectal cancer. Veterans Affairs Cooperative Study Group 380. N Engl J Med 2000;343(16):162-8.

34. Bond JH. Colon polyps and cancer. Endoscopy 2003;35(2): 27-35.

35. Winawer SJ, Fletcher RH, Miller L, Godlee F, Stolar MH, Mulrow CD, et al. Colorectal cancer screening: clinical guidelines and rationale. Gastroenterology 1997;112(2): 594-642.

36. Winawer SJ. Natural history of colorectal cancer. Am J Med 1999;106:3S-6S.

37. Schulmann K, Reiser M, Schmiegel W. Colonic cancer and polyps. Best Pract Res Clin Gastroenterol 2002;16(1): 91-114.

38. Seitz U, Bohnacker S, Seewald S, Thonke F, Brand B, Braiutigam $\mathrm{T}$, et al. Is endoscopic polypectomy an adequate therapy for malignant colorectal adenomas? Presentation of 114 patients and review of the literature. Dis Colon Rectum. 2004;47(11):1789-96.

39. Winawer SJ, Zauber AG, Ho MN, O’Brien MJ, Gottlieb LS, Sternberg SS, et al. Prevention of colorectal cancer by colonoscopic polypectomy. The National Polyp Study Workgroup. N Engl J Med. 1993;329(27):1977-81.

\section{Endereço para correspondência:}

Fábio Ramos Teixeira

Av. Oceânica, 1077, Condomínio Corais

Atalaia - Aracaju (SE), Brasil

CEP 49035-000

Tel.: (79) 9937-0886

E-mail: ramos15@superig.com.br 
Anexo 1 - Protocolo de pesquisa

Pólipos e lesões plano-elevadas colorretais

\begin{tabular}{|c|c|c|}
\hline \multicolumn{2}{|l|}{ Nome: } & \multirow{2}{*}{$\begin{array}{l}\text { Registro: } \\
\text { Gênero: ( ) M ( ) F }\end{array}$} \\
\hline Idade: & nento: & \\
\hline Estado civil: & \multicolumn{2}{|l|}{ Profissão: } \\
\hline Procedência: & \multicolumn{2}{|l|}{ Naturalidade: } \\
\hline \multicolumn{3}{|l|}{ Endereço: } \\
\hline \multicolumn{2}{|l|}{ Data do exame: } & Cor: \\
\hline \multicolumn{3}{|c|}{ Indicação colonoscópica: } \\
\hline \multicolumn{3}{|c|}{ Colonoscopia ( ) Retossigmoidoscopia ( ) } \\
\hline 1) $\mathrm{N}^{\mathrm{o}}$ de pólipos visualizados: $\quad$ Quantos excisados: & \multicolumn{2}{|l|}{ Quantos excisados: } \\
\hline \multicolumn{3}{|l|}{ 2) Diâmetro(s): } \\
\hline 3) Aspecto macroscópico: & \multicolumn{2}{|l|}{ 4) Localização: } \\
\hline $\begin{array}{l}\text { ( ) séssil } \\
\text { ( ) pediculado } \\
\text { ( ) lesão plano-elevada }\end{array}$ & $\begin{array}{l}\text { ( ) Ceco } \\
\text { ( ) Cólon ascendente } \\
\text { ( ) Flexura hepática } \\
\text { ( ) Cólon transverso } \\
\text { ( ) Flexura esplênica }\end{array}$ & $\begin{array}{l}\text { ( ) Cólon descendente } \\
\text { ( ) Cólon sigmóide } \\
\text { ( ) Retossigmóide } \\
\text { ( ) Reto } \\
\text { ( ) não informado }\end{array}$ \\
\hline \multicolumn{3}{|l|}{ Diagnóstico(s) associado(s): } \\
\hline Anatomapatológico: ( ) sim & \multicolumn{2}{|l|}{ ( ) não } \\
\hline \multicolumn{3}{|l|}{ Tipo histológico: } \\
\hline $\begin{array}{l}\text { 1) adenoma } \\
\text { ( ) tubular } \\
\text { ( ) túbulo-viloso } \\
\text { ( ) viloso } \\
\text { Grau de displasia: } \\
\text { ( ) Baixo grau } \\
\text { () Alto grau }\end{array}$ & $\begin{array}{l}\text { 2) não-adenoma } \\
\text { ( ) hiperplásico } \\
\text { ( ) inflamatório } \\
\text { ( ) lipoma } \\
\text { ( ) hamartoma } \\
\text { ( ) outro }\end{array}$ & $\begin{array}{l}\text { 3) lesão maligna } \\
\text { ( ) adenocarcinoma } \\
\text { ( ) tumor carcinóide } \\
\text { ( ) outro }\end{array}$ \\
\hline
\end{tabular}

